\title{
Development of Printed Sensors for Taste Sensing
}

\author{
Anindya Nag, Student Member, IEEE, Subhas Mukhopadhyay, Fellow, IEEE and Jürgen Kosel
}

\begin{abstract}
The paper presents an idea of developing taste sensors using novel printed sensors. The raw materials used for developing the sensors were commercial polymer films. Powered graphene was produced using laser induction technique. This powder was separately transferred to Kapton tapes to developed flexible graphene sensors. The fabricated sensors were tested with different chemicals having specific attributes with the idea to develop a taste sensor. Three different types of chemicals were tested and analyzed to verify the ability of the developed sensor patch to differentiate between the individual chemicals. The initial results have provided a significant platform in the process of developing a fully functionalized taste sensing system.
\end{abstract}

\section{INTRODUCTION}

The prominent use of sensors since the last two decades $[1,2]$ have revolutionized the thinking approach of human beings. Nowadays, sensors have been used in almost every sector for monitoring and analytical purposes. Environmental monitoring [3-5], detection of constituents in food and beverages [6-8], physiological parameters [9-11], are some of the most common applications of sensors in the current world. Apart from this, one of the common problems confronted by people is the loss of taste buds[12]. Salivary gland infection, gastroesophageal reflux disease, poor dental hygiene are some of the reasons for this occurrence. This could be a threat to the affected person with the likelihood of stroke, diabetes and heart diseases. This is especially seen among middle-aged and elderly people [13, 14]. Work to develop taste sensors had been done [15-19] previously by different research groups. But, there were certain disadvantages in those proposed techniques which drove us to consider alternative approaches. Some of the developed sensors [16] involved coated surface area which would be selective towards specifically tested chemicals. Now, this might be highly sensitive towards laboratory-based experiments, but it cannot be tested in real time applications. Also, there would be no re-use ability of the same sensor as the sensing surface would have to be re-coated every time experiments are done with it. Some of the sensors involved rigid substrates like glass would make it impossible to use it in real-time applications [15]. Some of the devices [17] did have flexible hybrid structures but were used to taste only one type of taste like bitter, which makes it insufficient for the sensor to distinguish between different tastes. So, it is a state-of-art to develop a low-cost sensor which can be easily fabricated and used in real-time applications. This paper

Anindya Nag and Prof. Subhas Mukhopadhyay are with Faculty of Science and Engineering, Macquarie University, Sydney, Australia. (Email: anindya1991@gmail.com, subhas.mukhopadhyay@mq.edu.au).

Prof. Jürgen Kosel is with Computer Electrical and Mathematical Sciences and Engineering Division, King Abdullah University of Science and Technology, Saudi Arabia (Email: jurgen.kosel@kaust.edu.sa). presents the design and fabrication of low-cost, flexible sensors [20]which was subsequently used as a taste sensor. The functionality of these sensors if proven successful can lead to surgical implantation on people with non-functioning taste buds. The signal from the sensor can be transmitted to the brain to determine which of the taste bud hormone to be stimulated for that particular food.

Low-cost polyimide (PI) films [21] were considered as the processing material for its distinct advantages. High bendability, high mechanical and thermal resistance and good solvent resistance are some of its advantages due to which it was preferred over other common flexible substrate materials like PDMS [22], PET [23], PEN [24]. Graphene was used as the conductive material to develop the electrodes. It is an allotrope of carbon which is the thinnest and strongest known till date. It is considered large for sensing materials due to very high conductivity towards heat and electricity. The Kapton tape used as the substrates for transferred graphene is preferred over other materials due to its low cost, high dielectric strength, and flexibility. Experiments were conducted with different chemicals to examine the change in sensor response towards them. The chemicals were considered according to their taste which would be similar to the different kinds of food people take daily. The responses were noted to analyze the functionality of the sensor patch to differentiate among the tested chemicals.

\section{FABRICATION OF PRINTED SENSORS}

The schematic diagram for individual steps performed to develop the sensor patches is shown in figure 1. Initially, the polyimide film was fixed to a glass substrate before it is taken to the laser platform. Universal laser systems using $\mathrm{CO}_{2}$ technology were used to curve the electrodes on the attached film. Interdigitated electrodes were designed on designing software (Corel DRAW) associated with the system. Before the laser induction process, the laser settings were specified accordingly. Power (Watt), speed $(\mathrm{m} / \mathrm{min})$ and $\mathrm{z}$-axis $(\mathrm{mm})$ are the three parameters that were altered and optimized to during the induction process. Power is the amount of energy of the emitted light from the nozzle. Speed determined the rate at which the nozzle moved over the processed material. Z-axis is the height of the laser platform from the laser nozzle during the process. The optimized values considered for fabrication was Power: 9 Watts, Speed: $70 \mathrm{~m} / \mathrm{min}$ and z-axis: $1 \mathrm{~mm}$. Powered graphene was developed when after the cutting was done on the attached film. 


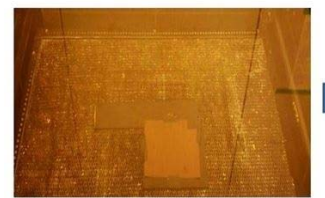

1. Polyimide film as template

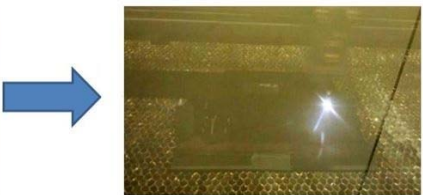

2. $\mathrm{CO}_{2}$ laser cutting of the polyimide film

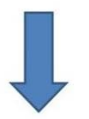

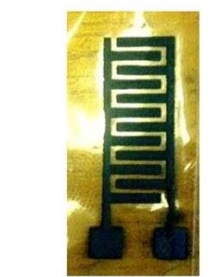

4. Transfer conductive material as sensor patch
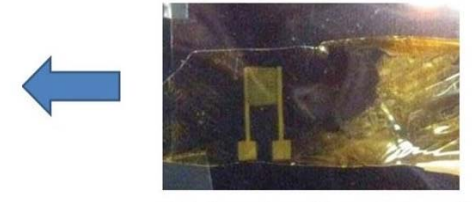

3. Transfer of laser induced graphene on Kapton
Fig. 1: Schematic diagram of the different fabrication steps performed to develop the sensor patches.

The shape of the cluttered graphene powder was in an interdigitated shape which was designed with the software. The design of the electrodes was interdigitated with six pairs of electrodes having a length and width of 500 microns and 100 microns respectively. The conductivity of the induced graphene was very high $(>50 \Omega)$. The graphene powder was transferred to Kapton tapes. This transfer was done by applying pressure manually on the Kapton tapes placed over the induced graphene. The placement of the tapes was done carefully for proper transfer of the graphene powder.

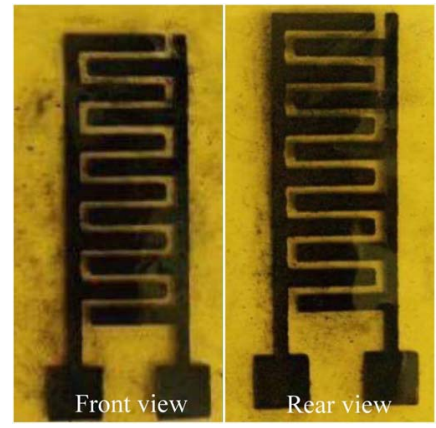

Fig. 2: Front and view of the developed sensor patches.

The graphene along on the Kapton tape was used a sensor patch for experimental purposes. Figure 2 depicts the front and rear view of the sensor patch. The difference in conductivities between the thermally-induced graphene and transferred graphene was less than $200 \Omega$.

\section{EXPERIMENTAL SET-UP}

Interdigital electrodes are shaped in a finger-like pattern which works on the principle of capacitive sensing. The structure made planar for non-invasive, one-directional measurement of different materials. The two electrodes of opposite polarity are called the excitation and sensing electrodes. When a time-dependent voltage is provided to the excitation voltage, an electric field is generated cause a shift in charge between the two plates. Due to the planar structure of the sensor, the electric field bulges out from one electrode to another. If any material is kept nearby or contacts with the sensing surface, the field penetrates through the material which changes its properties. Figure 3 represents the schematic diagram of the working principle of the interdigital sensor. L1, L2, and L3 are the differences in penetration depth of the electric field on the material under test (MUT). The height of the penetration depth can be adjusted by varying the inter-electrode distance. The change in a property of the field is analyzed by different impedimetric techniques to determine the characteristics of the MUT.

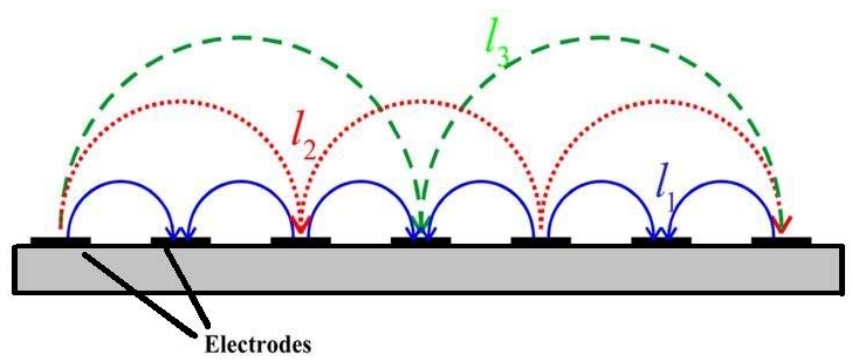

Fig. 3: Working principle of the interdigital sensor.

Electrochemical spectroscopy (EIS) was the technique used to perform the experiments with this sensor. EIS is a noninvasive technique where an electrochemical cell is studied over a range of frequencies to determine its response to impedance. Even though the impedance $(Z)$ consists of real and imaginary parts being the resistance (R) and the reactance $(\mathrm{X})$ respectively, the reactance is studied preferably due to its dependence on frequency $(X=$ $-1 / j * 2 * \pi * f * C)$. But in complex systems, both the resistance and reactance changes with frequency, with the later one changing predominantly more than the former one. The experimental results shown in this paper are in terms of reactance $(\mathrm{X})$ and phase angle $(\Phi)$ with respect to frequency. The phase angle $(\Phi)$ is the angle between the input voltage and output current. The experiments were performed with a HIOKI IM 3536 LCR Hi precision tester. Because of the flexibility of the sensor patch, it was attached to a wiring board by biocompatible tapes. This maintained the stability of the sensor inside the solutions during experimentation. Figure 4 shows the attached patch on the wiring board. The electrodes of the sensor were connected to Kelvin probes which were in turn connected to the LCR meter by BNC cables.

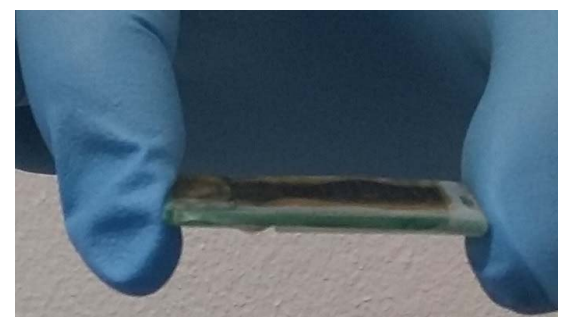

Fig. 4: Attachment of the sensor patch to the wiring board for experimental purposes. 
The testing of the sensor was done by forming aqueous solutions with different chemicals. Salty and sour testing was done by mixing sodium chloride $(\mathrm{NaCl})$ and citric acid $\left(\mathrm{C}_{6} \mathrm{H}_{8} \mathrm{O}_{7}\right)$ in water at definite proportions. De-ionized water (Resistance: 18.2 M $\Omega$ ) was considered for experimental purposes due to the minimized presence of minerals and ions in it. A series of solutions were formed from $5 \mathrm{ppm}$ to 30 $\mathrm{ppm}$ with a gap of $5 \mathrm{ppm}$. A stock solution of $100 \mathrm{ppm}$ was made by mixing $0.1 \mathrm{mg}$ of solute and $1000 \mathrm{ml}$ of solvent. This was thoroughly mixed with a magnetic stirrer for 25 mins before using it to form the testing solutions. A solution of $5 \mathrm{ppm}$ was made by mixing $5 \mathrm{ml}$ of stock solution with 95 $\mathrm{ml}$ of de-ionized water. This was followed by mixing $10 \mathrm{ml}$ of stock solution with $90 \mathrm{ml}$ of de-ionized water to form 10 ppm solution. A frequency sweep between $1 \mathrm{KHz}-10 \mathrm{KHz}$ was done to test the change in response of the sensor. Three readings were considered per experiment to be taken as an average to validate the repeatability in the response of the sensor. After the testing with one solution, the sensor was washed thoroughly with water and dried in the oven for 15 minutes before it for testing with the next solution.

\section{EXPERIMENTAL RESULTS}

Figures 5-6 show the response of the sensor for experiments done with citric acid solutions. As can be seen, the change in phase angle $(\Phi)$ is more significant compared to reactance $(\mathrm{X})$. The change in reactance is very small for higher concentrations as shown in the inset in figure 4. The sensitive region is between $5.63 \mathrm{KHz}-10 \mathrm{KHz}$ where the difference in results for different concentrations can be made distinctly. Figures 7-8 show the experimental results done with sodium chloride for sour testing purposes. It is seen that the sensitive region of both the citric acid and sodium chloride is same. But there has been a sharp difference among the range in impedance and phase angle between the two chemicals. The ranges are given in Table 1 . This shows that the two chemicals can be distinguished based on their readings. Any daily food taken by a person that falls under these two categories can be differentiated based on the response given by the sensor in the absence of the taste buds. It would be understood that any food tested with this sensor would fall outside these two categories if the response happens to be outside these given ranges.

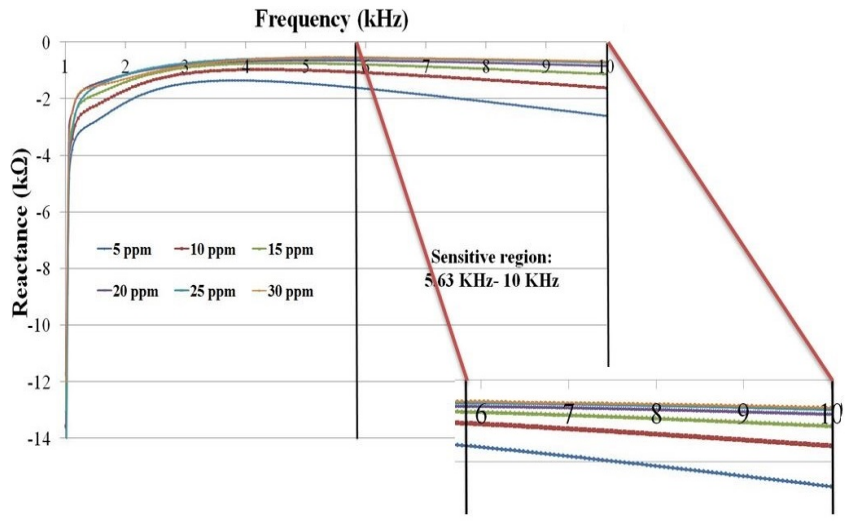

Fig. 5: Response of the sensor for the experiments done with citric acid in terms of reactance on frequency.

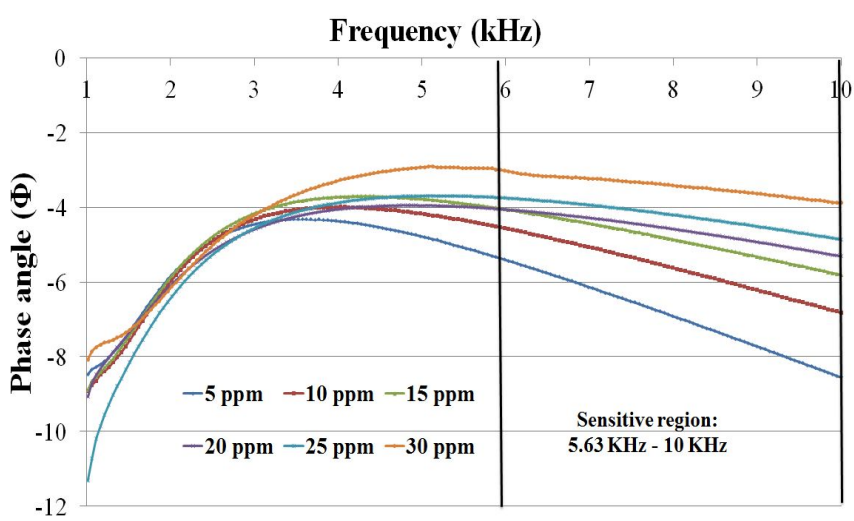

Fig. 6: Response of the sensor for the experiments done with citric acid in terms of phase angle on frequency.

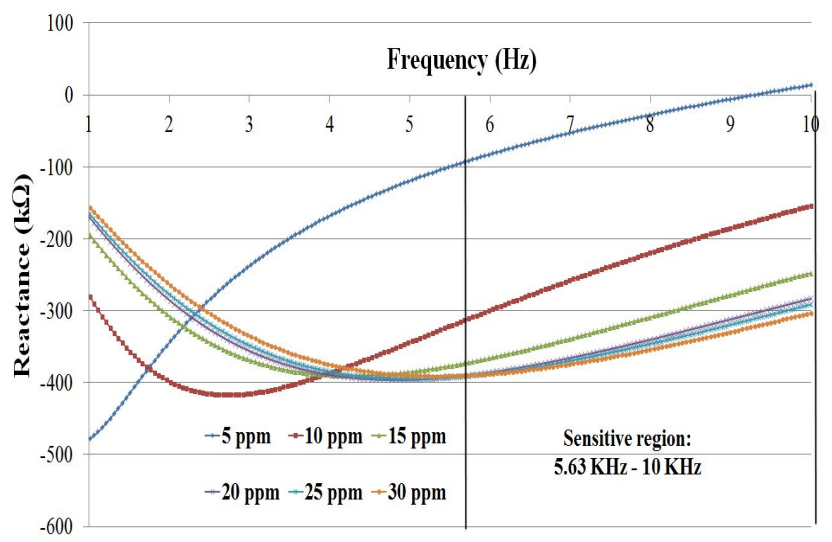

Fig. 8: Response of the sensor for the experiments done with sodium chloride in terms of phase angle on frequency.

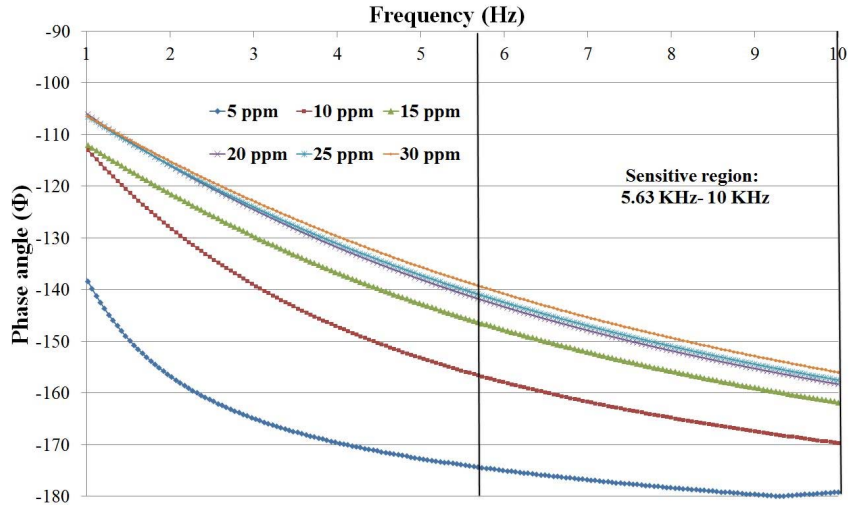

Fig. 7: Response of the sensor for the experiments done with sodium chloride in terms of phase angle on frequency.

TABLE 1. RANGE OF VALUES REGARDING REACTANCE AND PHASE ANGLE FOR THE TESTED CHEMICALS.

\begin{tabular}{|l|l|l|}
\hline \multicolumn{1}{|c|}{ Chemical } & \multicolumn{1}{c|}{$\begin{array}{c}\text { Range in reactance values } \\
(\mathbf{k} \boldsymbol{\Omega})\end{array}$} & $\begin{array}{c}\text { Range in phase } \\
\text { angle }(\boldsymbol{\Phi})\end{array}$ \\
\hline Citric acid & $(-669)-(-4040)$ & $(-8.06)-(-11.3)$ \\
\hline $\begin{array}{l}\text { Sodium } \\
\text { chloride }\end{array}$ & $(-1570)-(-4780)$ & $(-106.4)-(-136.3)$ \\
\hline
\end{tabular}




\section{CONCLUSION}

The experimental results shown above validate the capability of the developed sensor of being used as a taste sensor. It does differentiate among the different chemicals which have a similar taste to some of the daily used food. The change is analyzed regarding the change in reactance $(\mathrm{X})$ and phase angle $(\Phi)$ on frequency. There are still some issues that need to be addressed before approaching towards the next step. Even though the chemicals tried in these experiments do resemble some of the daily food, there are variations in the taste of a similar kind of taste which causes variations in the response. More experiments with varied parameters like $\mathrm{pH}$ of that chemical would be conducted to have a detailed analysis. Also, the response of the sensor for different concentrations in the case of sour chemical testing is very low. A signaling circuit would be associated with the sensor before it is processed for monitoring purposes. These points would be taken into consideration en route in developing a fully functionalized system.

\section{ACKNOWLEDGMENT}

The research reported in this paper has been assisted by King Abdullah University of Science and Technology (KAUST), Saudi Arabia, for providing the facilities to fabricate the sensor patches. They would also thank Macquarie University, Australia for providing the optimum laboratory conditions for performing the experiments.

Ethics approval was not required for the conducted experiments or the reported experimental results. This is because the experiments were performed in aqueous without the involvement of any living organism.

\section{REFERENCES}

[1] L. E. Foster, Nanotechnology: science, innovation, and opportunity: Prentice Hall PTR, 2005.

[2] A. Ahammad, J.-J. Lee, and M. A. Rahman, "Electrochemical sensors based on carbon nanotubes," Sensors, vol. 9, no. 4, pp. 2289-2319, 2009.

[3] A. Nag, A. I. Zia, X. Li et al., "Novel Sensing Approach for LPG Leakage Detection: Part I-Operating Mechanism and Preliminary Results," IEEE Sensors Journal, vol. 16, no. 4, pp. 996-1003, 2016.

[4] A. Nag, A. I. Zia, X. Li et al., "Novel Sensing Approach for LPG Leakage Detection-Part II: Effects of Particle Size, Composition, and Coating Layer Thickness," IEEE Sensors Journal, vol. 16, no. 4, pp. 1088-1094, 2016.

[5] A. Nag, and S. Mukhopadhyay, "Smart Home: Recognition of activities of elderly for 24/7; Coverage issues," Proceedings of the 2014 International Conference on Sensing Technology, Liverpool, U.K., 2014, pp. 480-489.

[6] A. I. Zia, A. Mohd Syaifudin, S. Mukhopadhyay et al., "Sensor and instrumentation for progesterone detection," Instrumentation and Measurement Technology Conference (I2MTC), IEEE International, Graz Austria, 2012, pp. 1220-1225.

[7] A. I. Zia, S. Mukhopadhyay, I. Al-Bahadly et al., "Introducing molecular selectivity in rapid impedimetric sensing of phthalates," Instrumentation and Measurement Technology Conference (I2MTC), IEEE International, Montevideo, Uruguay, pp. 838-843.

[8] A. I. Zia, M. S. A. Rahman, S. C. Mukhopadhyay et al., "Technique for rapid detection of phthalates in water and beverages," Journal of Food Engineering, vol. 116, no. 2, pp. 515-523, 2013.

[9] A. Nag, S. Mukhopadhyay, and J. Kosel, "Transparent biocompatible sensor patches for touch sensitive prosthetic limbs." pp. 1-6.
[10] A. Nag, S. C. Mukhopadhyay, and J. Kosel, "Flexible carbon nanotube nanocomposite sensor for multiple physiological parameter monitoring," Sensors and Actuators A: Physical, vol. 251, pp. 148$155,2016$.

[11] A. Nag, S. C. Mukhopadhyay, and J. Kosel, "Tactile Sensing From Laser-Ablated Metallized PET Films," IEEE Sensors Journal, vol. 17, no. 1, pp. 7-13, 2016.

[12] "Impaired Taste," http://www.healthline.com/health/taste-impaired. Last accessed on 17.10.2017.

[13] "Loss of taste buds in elderly people," http://www.everydayhealth.com/senior-health/when-aging-stealsyour-sense-of-taste.aspx. Last accessed on 17.10.2017.

[14] "Taste disorders," https://www.nidcd.nih.gov/health/taste-disorders Last accessed on 17.10.2017.

[15] Q. Liu, F. Zhang, D. Zhang et al., "Extracellular potentials recording in intact taste epithelium by microelectrode array for a taste sensor," Biosensors and Bioelectronics, vol. 43, pp. 186-192, 2013.

[16] Y. Tahara, A. Ikeda, Y. Maehara et al., "Development and evaluation of a miniaturized taste sensor chip," Sensors, vol. 11, no. 10, pp. 9878-9886, 2011.

[17] T. H. Kim, H. S. Song, H. J. Jin et al., “"Bioelectronic super-taster” device based on taste receptor-carbon nanotube hybrid structures," Lab on a Chip, vol. 11, no. 13, pp. 2262-2267, 2011.

[18] J. K. Lorenz, J. P. Reo, O. Hendl et al., "Evaluation of a taste sensor instrument (electronic tongue) for use in formulation development," International journal of pharmaceutics, vol. 367, no. 1, pp. 65-72, 2009.

[19] Y. Tahara, K. Nakashi, K. Ji et al., "Development of a portable taste sensor with a lipid/polymer membrane," Sensors, vol. 13, no. 1, pp. 1076-1084, 2013.

[20] A. Nag, S. C. Mukhopadhyay, and J. Kosel, "Sensing System for Salinity Testing Using Laser-induced Graphene Sensors," Sensors and Actuators A: Physical, vol. 264, pp. 107-116, 2017.

[21] J. A. Dobrzynska, and M. A. Gijs, "Flexible polyimide-based force sensor," Sensors and Actuators A: Physical, vol. 173, no. 1, pp. 127 135,2012

[22] J.-H. Moon, D. H. Baek, Y. Y. Choi et al., "Wearable polyimidePDMS electrodes for intrabody communication," Journal of Micromechanics and Microengineering, vol. 20, no. 2, pp. 025032 , 2010.

[23] M.-x. Jing, C. Han, M. Li et al., "High performance of carbon nanotubes/silver nanowires-PET hybrid flexible transparent conductive films via facile pressing-transfer technique," Nanoscale research letters, vol. 9, no. 1, pp. 1-7, 2014.

[24] R. Shabannia, and H. Abu-Hassan, "Vertically aligned $\mathrm{ZnO}$ nanorods synthesized using chemical bath deposition method on seed-layer $\mathrm{ZnO}$ /polyethylene naphthalate (PEN) substrates," Materials Letters, vol. 90, pp. 156-158, 2013 